\title{
Data-driven Demand Response Characterization and Quantification
}

\author{
Le Ray, Guillaume; Pinson, Pierre; Larsen, Emil Mahler
}

Published in:

Proceedings of 12th IEEE Power and Energy Society PowerTech Conference

Publication date:

2017

Document Version

Peer reviewed version

Link back to DTU Orbit

Citation (APA):

Le Ray, G., Pinson, P., \& Larsen, E. M. (2017). Data-driven Demand Response Characterization and

Quantification. In Proceedings of 12th IEEE Power and Energy Society PowerTech Conference IEEE.

\section{General rights}

Copyright and moral rights for the publications made accessible in the public portal are retained by the authors and/or other copyright owners and it is a condition of accessing publications that users recognise and abide by the legal requirements associated with these rights.

- Users may download and print one copy of any publication from the public portal for the purpose of private study or research.

- You may not further distribute the material or use it for any profit-making activity or commercial gain

- You may freely distribute the URL identifying the publication in the public portal

If you believe that this document breaches copyright please contact us providing details, and we will remove access to the work immediately and investigate your claim. 


\section{Data-driven Demand Response Characterization and Quantification}

\author{
Guillaume Le Ray, Pierre Pinson \\ Technical University of Denmark \\ Centre for Electric Power and Energy \\ Kgs. Lyngby, Denmark \\ email: \{gleray, ppin\}@elektro.dtu.dk
}

\author{
Emil Mahler Larsen \\ Danish Energy Association \\ Frederiksberg, Denmark \\ email: eml@danskenergi.dk
}

\begin{abstract}
Analysis of load behavior in demand response (DR) schemes is important to evaluate the performance of participants. Very few real-world experiments have been carried out and quantification and characterization of the response is a difficult task. Nevertheless it will be a necessary tool for portfolio management of consumers in a DR framework. In this paper we develop methods to quantify and characterize the amount of DR in a load. The contribution to the aggregated load from each household is quantified on a daily basis, showing the potential variability of the response in time. Clustering on the average values and standard deviation of the contribution regroups households with the same average response. Independent Component Analysis (ICA) is used to characterize different DR delivery profiles.
\end{abstract}

Index Terms-Demand Response (DR), DR characterization, DR quantification, smart grid, energy analytics.

\section{INTRODUCTION}

Research and the application of controlling electric loads in useful ways continues to grow as system operators look for new ways to balance production when a large share of renewable energy sources (RES) is present. Demand Response (DR) answers this problem by moving the load in time or shedding it. DR's rise has coincided with large-scale smart meter roll-outs across the world, which provide more precise information about the demand status. Utilities often consider two applications of DR: emergency use, which is a safety net in case of unexpected outages, and economic, which reduces the use of energy-only ancillary services [1].

Different DR schemes have been designed and implemented, with wide-ranging benefits and drawbacks, although how to quantify DR during real operation remains underexplored [2], [3]. Indirect control through dynamic tariffs has emerged as a popular option due to its ability to respond quickly to variation in production (i.e. wind, solar) and the simplicity of financial settlement, i.e. reward for consumers providing flexibility [4]. Indirect control does not require each load to respond to price signals in the same way, but the population's response as a whole remains nevertheless statistically predictable.

The evaluation of how much DR is in a load is often made by comparison to a baseline. The baseline can be generated using a model fitted on consumption data from historical nonDR days [5]. It can also be simultaneously collected from a reference sample of households which has the same characteristics (i.e. number, size, temperature) as the DR sample of households but which do not receive DR incentives [6]. However, sufficient data to generate reliable forecasts of loads or representative reference set are not always available. In the literature, little work has been done on quantifying and characterizing flexible loads from residential consumers in a DR framework when a baseline is not available.

In this paper we propose: 1) a methodology to retrospectively evaluate how much DR was delivered, on average, in a group of similar loads without estimating a baseline; 2) a method which gives information about the characteristics of the response in terms of amplitude and rate of response, i.e. how large the response is and how often a load is able to deliver the response. When managing a portfolio of households in a DR framework, it is the combination of both quantification and characterization of the response that is important when generating the future prices. Furthermore, combining the average response from groups of consumers, each receiving a different price signal, can then be a possible way to obtain a desirable aggregated load.

The paper is organized as follows: Section II presents the data and the EcoGrid EU DR implementation; Section III introduces the methodology to categorize, characterize and quantify the response to price; Section IV presents the results of the method applied to data from EcoGrid EU and Section V provides conclusions and ideas for future work.

\section{EMPIRICAL FRAMEWORK}

The dataset used comes from the EcoGrid EU project, which was completed in 2015. The goal of EcoGrid EU was to exploit the flexibility of residential consumers in a large scale realtime market with a large share of RES in the energy mix [7].

\section{A. Hypothesis on quantifying real time pricing DR}

Houses are equipped with automated devices from different manufacturers, which in turn can be controlled by different algorithms. Each house has its own threshold for which it reacts to price, according to the comfort settings determined by the family living there. We suppose that the controller's algorithm does not affect the amplitude of the response but the pace and the frequency at which the response is delivered. 
The behavior of a load is dynamic and depends on the household activity. It is therefore stochastic and should be analyzed as such. Each house may respond differently from one day to another, depending on the activity of the inhabitants. The categorization of the house's DR delivery should therefore be based on both the average response and its variability from day-to-day, since the latter describes the reliability of a portfolio.

\section{B. Real Time Pricing Market Presentation}

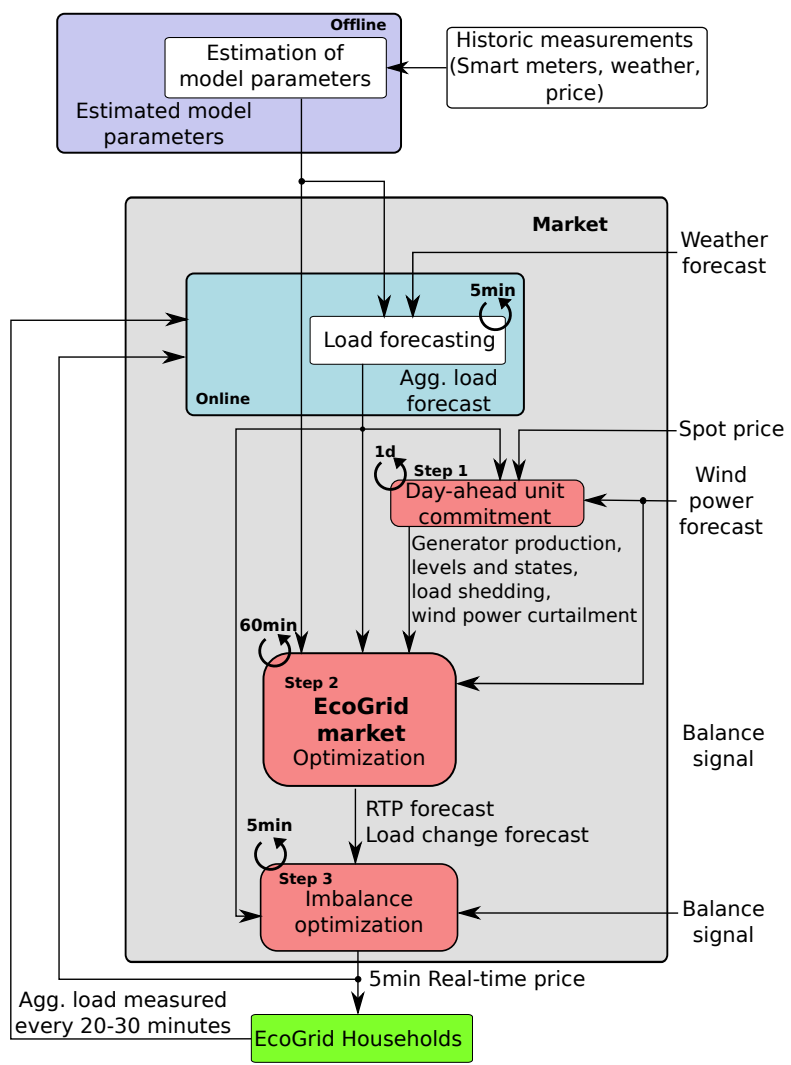

Fig. 1: Overall representation of EcoGrid EU market and price generation.

The DR framework in EcoGrid EU is a hardware-in-theloop market platform (Figure 1). The market design can be divided into four blocks:

1) The forecasting module computes the load forecast based on historical consumption, weather forecasts prices.

2) The Day-ahead unit commitment schedules generation according to known spot prices and the wind power forecast.

3) The EcoGrid market is the core market clearing that finds 5-minute prices for the next hour. Its main objective is to maintain the system balance and maximize the social welfare by dispatching conventional balancing power and DR (via 5-minute prices).

4) The 5-min imbalance optimization makes minor adjustment to prices before committing the real-time price.
The Real-Time Price (RTP) generally follows the daily load pattern, since the spot price (a component of the RTP) is positively correlated with the consumption profile - periods of high demand cause a higher spot price. When a load is reactive, the consumption moves in an opposite direction to the price variation (i.e. consumption increases as the price decreases and vice versa). A description of the market clearing mechanism that determines the real-time price is described in [8].

\section{Electric Load Data Collection}

EcoGrid EU relied mostly on heating devices that could deliver DR by exploiting the implicit thermal storage of households. Out of 1900 houses in total, the houses with good quality data are listed in Table I. There were four groups that were fitted with automated equipment (groups 3-6). group 1 was designed as a reference group, but was shown to be non representative when attempting to generate a reliable baseline [9]. group 2 was a manual group with no automation of devices and participants were asked to modify their consumption through SMS. Two types of heating were installed in the other households: Electric Heating $(\mathrm{EH})$ or Heat Pump (HP); the equipment (i.e. controller) installed in the households of groups 3-5 comes from the same manufacturer, with different algorithms for group 5. Group 6 is equipped with a different hardware and software. The data was collected from September 2014 to February 2015 with temperature spanning from $-10.6{ }^{\circ} \mathrm{C}$ to $18.0{ }^{\circ} \mathrm{C}$. The resolution of the electric load is 5 minutes, with data transferred to a central database every 20-30 minutes. Houses and time periods with more than $10 \%$ values missing were filtered out.

TABLE I: TEST GROUP DESCRIPTIONS.

\begin{tabular}{lcccc}
\hline Group & type of heating & manufacturer & type of DR & number \\
\hline Group 1 & mixed & none & none & 253 \\
Group 2 & mixed & none & manual & 455 \\
\hline Group 3 & $\mathrm{HP}^{a}$ & manuf. 1 & auto. & 195 \\
Group 4 & $\mathrm{EH}^{b}$ & manuf. 1 & auto. & 322 \\
Group 5 & $\mathrm{HP}$ & manuf. 2 & auto. & 84 \\
Group 6 & $\mathrm{EH}$ & manuf. 3 & auto. & 398 \\
\hline${ }^{a}$ Heat Pump & & & & \\
${ }^{b}$ Electric Heating & & & &
\end{tabular}

\section{Methodology fOR QUANTIFYING AND CHARACTERIZING RESPONSE TO RTP}

The behavior of a load can change from extremely reactive to not reactive from one day to another as the inhabitants can change the thermostat temperature settings (affecting the comfort zone by extension). We evaluate the DR delivered by each house on a daily basis and summarized it using the average and standard deviation per household rather than evaluating it over the entire heating season. 


\section{A. Response Categorization}

As previously described, the RTP follows the average daily pattern of the load. The less a load provides DR, the closer it will correlate with the RTP. This is captured by a positive correlation between a load $x_{n}$ and RTP $p$ as presented in (1). When a load is reactive to a price variation and show some $\mathrm{DR}$, the variation of the load is opposite to the price and the correlation between the load and RTP is then negative.

$$
\begin{gathered}
\operatorname{Cor}\left(p, x_{n}\right)=\frac{\sum_{t=1}^{T}\left(p_{t}-\bar{p}\right)\left(x_{n, t}-\overline{x_{n}}\right)}{\sqrt{\sum_{t=1}^{T}\left(p_{t}-\bar{p}\right)^{2}} \sqrt{\sum_{t=1}^{T}\left(x_{n, t}-\bar{x}\right)^{2}}} \\
d . \operatorname{Cor}\left(p, x_{n}\right)=\sqrt{\left(\frac{1-\operatorname{Cor}\left(p, x_{n}\right)}{1+\operatorname{Cor}\left(p, x_{n}\right)}\right)}
\end{gathered}
$$

Using the correlation-based distance (d.Cor) in (2) between the individual loads $x_{n}$ and the RTP $p$ of a single day, the response during that day can be quantified [10]. Note that an average correlation of 0 over a day corresponds to a d.Cor of one and the more negative the correlation is, the larger the d.Cor.

The d.Cor is calculated for each household and every day of the heating season. The mean and standard deviation is calculated per household in order to cluster them according to their average distance from the RTP and how it varies during the season. The cluster algorithm used to split the data is Kmeans $(\mathrm{k}=5)$ run with 25 different sets of starting points to validate the stability of the partition.

\section{B. Response Quantification}

The response in each cluster, is estimated using Finite Impulse Responses (FIR) for price [9]. FIRs are obtained from a general linear model of $x_{t}$ the observed load,

$$
x_{t}={\widetilde{\lambda_{t}}}^{\top} \theta_{\lambda}+{\widetilde{z_{t}}}^{\top} \theta_{z}+\widetilde{\chi}_{t}^{\top} \theta_{\chi}+\epsilon_{t}
$$

where $\widetilde{\lambda}_{t}^{\top}$ is a vector including forecast, real-time and historic electricity prices (e.g. day-ahead, hour-ahead and RTP), ${\widetilde{z_{t}}}^{\top}$ is a vector of exogenous variables (e.g. solar radiance, wind speed, exterior temperature, Fourier series of the daily independent base load), $\tilde{\chi}_{t}^{\top}$ is a vector of interactions of some of the precedent variables and $\epsilon_{t}$ is the normally distributed error.

The FIRs correspond to the vector of relative real-time price coefficients $\theta_{\lambda}$ in (3). It is calculated based on the relative price which is the difference, at each time step, between the RTP and the hour-ahead price. The fitting of the model is done using a Lasso penalization [11].

\section{Response Characterization}

The RTP exhibits many local extremii during a single day,to which households do not always react. Clustering is a good way to group the households according to their responses on average, but when more details are required to characterize the response, the limits of the method are reached. Each cluster is the average reaction of a pool of households and, although confidence intervals can be calculated, they obscure an otherwise describable, diverse set of behaviors. To characterize the loads, let us first consider that each consumption time-series is a linear combination of $I$ different consumption pattern $s_{i}$ and that the vector of time-series consumption $\boldsymbol{x}=\left\{x_{1}, \ldots, x_{n}, \ldots, x_{N}\right\}$ can be written,

$$
\boldsymbol{x}=\sum_{1}^{I} a_{i} s_{i}
$$

where $a_{i}$ is a vector of coefficients. Each component $a_{i}$ represents a response to the price or heating preferences setting and, by combining and weighting them, the consumption of the time-series can be reconstructed. The method is called Independent Component Analysis (ICA) [12]. It was first used in signal processing to solve the problem of blind source separation, like the cocktail party problem. (4) can also be written,

$$
\boldsymbol{x}=\boldsymbol{a s}
$$

where $\boldsymbol{a}=\left\{a_{1}, \ldots, a_{i}, \ldots, a_{I}\right\}$ is a matrix of coefficients and $\boldsymbol{s}=$ $\left\{s_{1}, \ldots, s_{i}, \ldots, s_{I}\right\}$ is a vector representing a base of synthetic signals with no units, called independent components (IC). After estimating $\boldsymbol{a}$, its inverse $\boldsymbol{w}$ is computed and the IC can be obtained by,

$$
s=\boldsymbol{w} \boldsymbol{x}
$$

and corresponds to weights of consumption time-series $\boldsymbol{x}$ on ICs $s$. The only assumptions made to perform an ICA are that the components should be statistically independent and so they must have non-gaussian distributions. The ICA estimation is based on measure of non-gaussianity (e.g. kurtosis, negentropy) of $w_{i}^{T} \boldsymbol{x}$ where $w_{i}^{T}$ is a transpose row $w_{i}$.

Before running an ICA, the signals are centered and whitened using Principle Component Analysis (PCA). Thus all signals have a zero mean and a unit variance.

The fastICA is used to perform the ICA and is presented in Algorithm 1 [12]. First $\boldsymbol{w}$ is initiated randomly by sampling from Gaussian distribution with $\mu=0$ and $\sigma^{2}=1$. A fixed point iteration process then finds the maximum non-gaussianity by approximating the negentropy using its approximative Newton iteration. In the presented implementation, the function $g$ and $g^{\prime}$ are respectively the derivative and second derivative of the non-quadratic function $G$,

$$
G(u)=-\exp \left(-u^{2} / 2\right)
$$

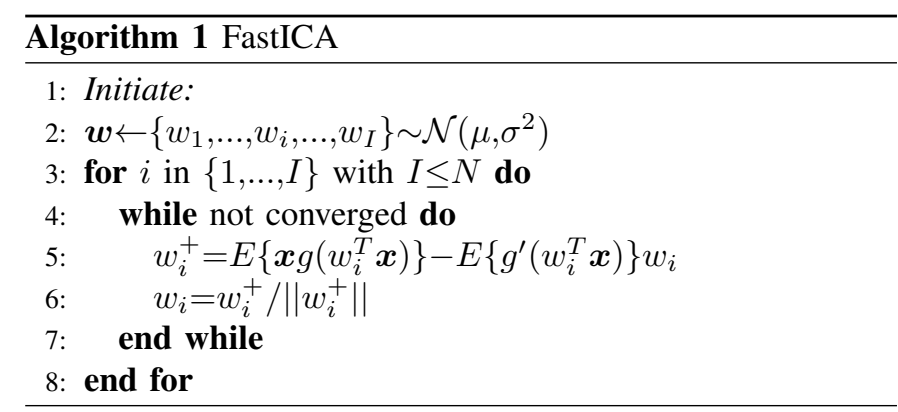




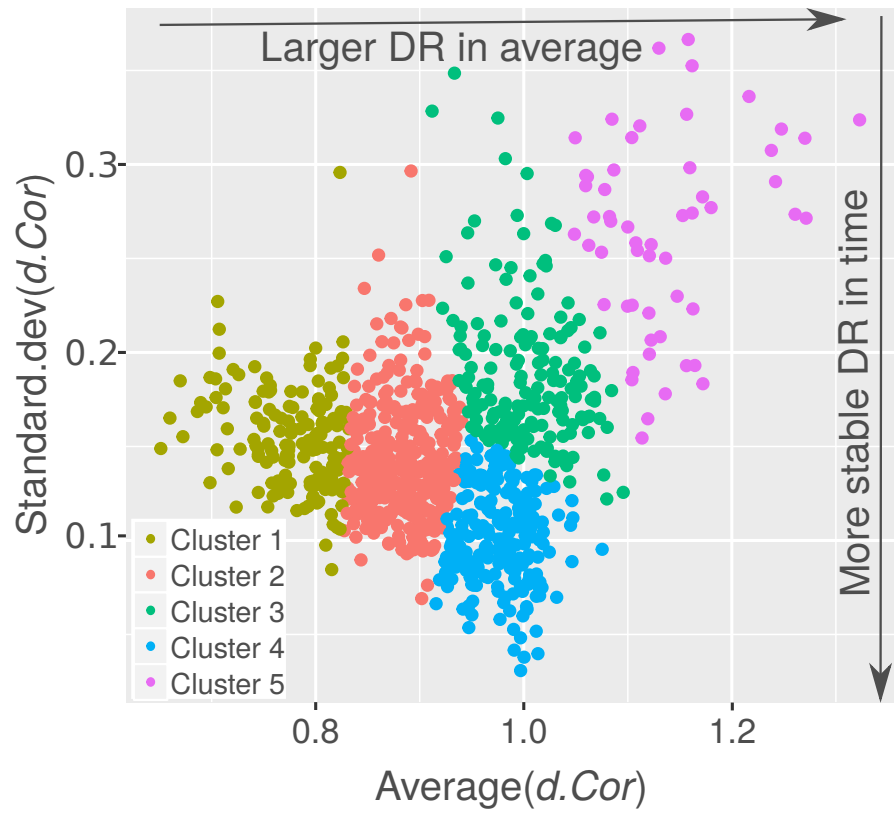

Fig. 2: Clusters generated with $\mathrm{K}$-means $(\mathrm{k}=5)$ on the mean and standard deviation of the d.Cor distance

\section{RESUlts}

\section{A. Response Categorization Results}

The results of the clustering are shown in Figure 2. Each point represents an household in the two dimensional space defined by the average d.Cor and the standard deviation of the d.Cor over the heating season. The color codes the partition into clusters. An increase of standard deviation with average d.Cor results from the non-linearity of d.Cor [10]. The standard deviation is mostly used to discriminate between houses with the same average participation but different variability over the heating season.

The analysis of repartition of the groups in each cluster (Figure 3 on the left) reveals that besides cluster 5, which is mostly composed of group 6 households, the manufacturer or the type of heating does not influence the average response. Indeed cluster 5 which is the farthest from the RTP, is mostly composed of households from the group 6 and 4 which are both equipped with EH but it could be the result of another factor as group 4 and 6 are also present in other clusters (e.g. cluster 3 ). With the exception of cluster 5 , these results appear to support the hypothesis that the group and subsequently the technology is not the main driver of the average response of the load as the groups are distributed in the different clusters.

The repartition of the dwelling types in each cluster is presented in Figure 3 on the right. The three clusters with the highest response to RTP on average present also the largest shares of holiday houses. On the one hand the fact that inhabitants are not present in the dwelling is a problem to d.Cor as the base load is then not following the base pattern of the price and it increases the value of the distance correlation. On the other hand the absence of interaction with inhabitants may also be an asset in the planning of the heating which
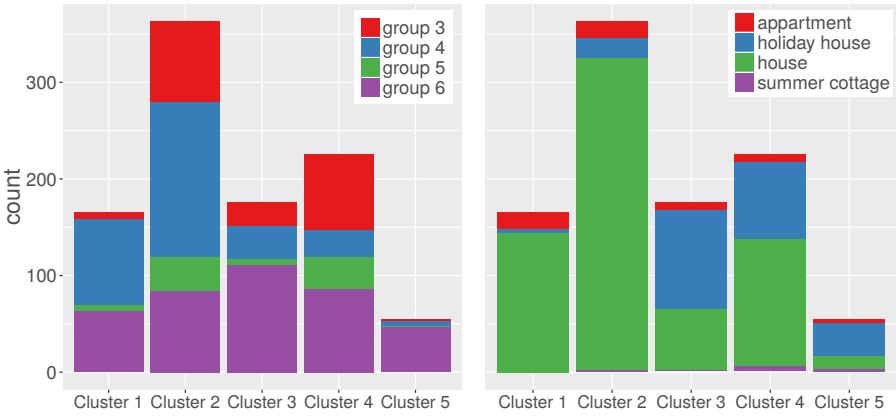

Fig. 3: Split of the groups (left) and the type of dwelling (right) into the K-means clusters

gives the controller the full control on planning the heating over a whole day.

\section{B. Response Quantification Results}

The quantification of the response has been done based on the clusters generated in Section IV-A. Figure 4 presents the FIR of each cluster, which is a traditional time-series analysis tool that represents the average response over the entire test period (not just a daily metric). The results are given in percentage of the maximum load.

With this traditional time-series analysis approach, cluster 4 exhibits the smallest response, peaking at $10 \%$ of the load 35 minutes after the price change, followed by a stiff and large rebound (21\%) where the energy to be recovered is actually larger than the initially delivery of energy. Cluster 4 shows a small increase in consumption when the change in price first arrives, which may be a time-delay caused by minimum runtimes, since this coincides with the slow DR delivery. Cluster 1 and 2 exhibit the next smallest DR volume, at $13 \%$, with a

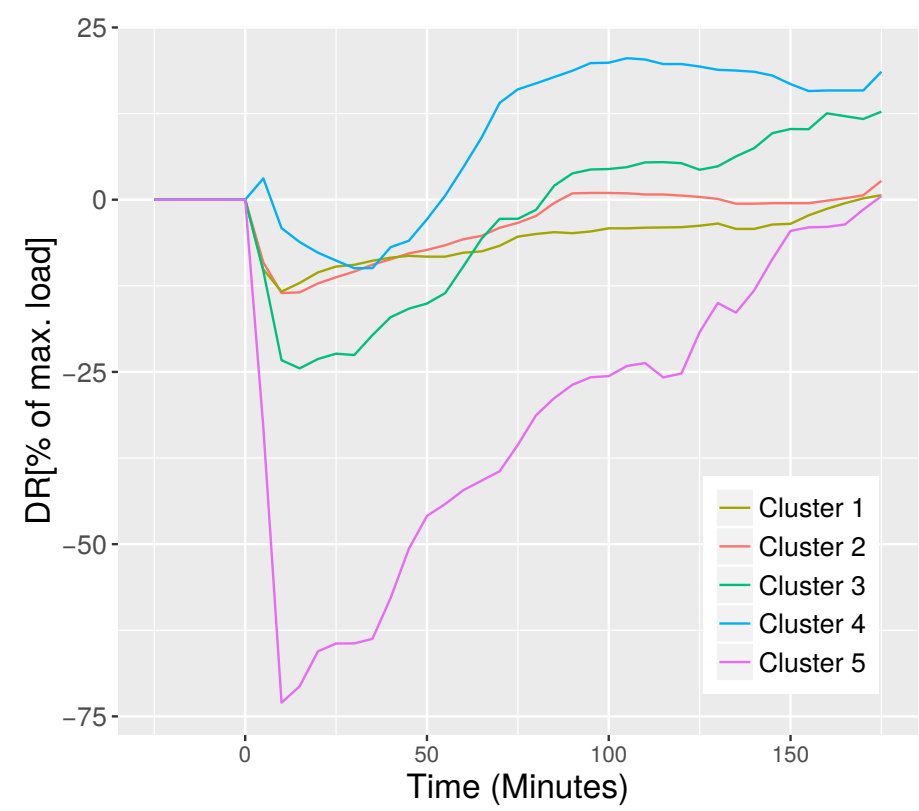

Fig. 4: FIR for the clusters obtained based on their average response and variability in the response. 
TABLE II: DISTRIBUTION OF THE GROUPS ABOVE THE $95 \%$ QUANTILE AND UNDER THE 5\% QUANTILE OF THE DENSITY OF WEIGHTS FOR EACH IC

(a) under the $5 \%$ quantile

\begin{tabular}{lcccccccccc} 
IC \# & 1 & 2 & 3 & 4 & 5 & 6 & 7 & 8 & 9 & 10 \\
\hline Group 3 & 33 & 5 & $\mathbf{1 0}$ & 5 & 14 & 4 & 0 & 5 & 4 & 5 \\
Group 4 & 12 & 21 & $\mathbf{2 9}$ & 7 & 16 & 21 & 0 & 10 & $\mathbf{2 7}$ & 23 \\
Group 5 & 2 & 4 & 4 & 1 & 7 & 3 & 14 & 7 & 2 & 4 \\
Group 6 & 3 & 20 & 7 & $\mathbf{3 7}$ & 13 & 22 & 36 & 28 & $\mathbf{1 7}$ & 18 \\
\hline
\end{tabular}

(b) above the $95 \%$ quantile

\begin{tabular}{lcccccccccc} 
IC \# & 1 & 2 & 3 & 4 & 5 & 6 & 7 & 8 & 9 & 10 \\
\hline Group 3 & 12 & 9 & 10 & 7 & 19 & 6 & 15 & 11 & 8 & 31 \\
Group 4 & 20 & 27 & 10 & $\mathbf{2 6}$ & 15 & 17 & 17 & 20 & $\mathbf{2 7}$ & 9 \\
Group 5 & 4 & 1 & 0 & 0 & 7 & 6 & 7 & 6 & 1 & 3 \\
Group 6 & 14 & 13 & $\mathbf{3 0}$ & 17 & 9 & 21 & 11 & 13 & $\mathbf{1 4}$ & 7 \\
\hline
\end{tabular}

long-lasting response $(85 \mathrm{~min}$. for cluster 2 and $175 \mathrm{~min}$. for cluster 1) followed by no significant rebound.

Cluster 3 delivers 24\% DR, with maximum delivery after 15 minutes. It exhibits a slow but larger rebound after 85 minutes.

The most homogeneous cluster, composed mostly of group 6 houses, is cluster 5, which delivers by far the most DR. Its DR amplitude reaches $72 \%$ of its maximum load, with a delay of just 10 minutes, signifying that this small cluster is composed of high performing houses. It does not present any rebound effect after the DR event. This result tentatively confirms the order of response from not much responsive (cluster 1) to large response (cluster 5) observed in Section IV-A.

\section{Response Characterization Results}

The ICA has been implemented on a single day; Tuesday the $6^{\text {th }}$ of January 2015. After whitening the consumption timeseries using PCA, the $I=10$ first principal components are kept to perform the ICA. The ten first components of the PCA collect $72 \%$ of the total variance of the data. The decomposition of the signals into ICs separates the independent observed behaviors of the loads. In other words, any load generated by a household (i.e. real or fictive) with the characteristics of the pool of households used to generate the ICs can be represented as a linear combination of the ICs as presented in (4). ICA projects each load pattern into a space with the ICs as a base of dimension $I$.

It is important to note that the ICA does not prioritize an IC over another when generation the base, which means that the ICs cannot be ranked according to a specific metric (e.g. $\%$ of explain variance in the case of PCA). The IC, when represented, corresponds to one possible direction (positive weights) of the base vector, which makes the interpretation non-direct. The extreme 5\% quantiles (above 95\% and under $5 \%$ ) of the density distribution of weights, which correspond to the most contributive points, are analyzed for each IC (Table II \& III) to evaluate which groups and which clusters contribute highly (positively or negatively) to each IC.

From Table II, Figure 5 and the knowledge gathered about the possible settings of the different equipment, an interpretation of the IC numbers 3, 4 and 9 is given.
TABLE III: DISTRIBUTION OF THE CLUSTERS ABOVE THE 95\% QUANTILE AND UNDER THE 5\% QUANTILE OF THE DENSITY OF WEIGHTS FOR EACH IC

(a) under the $5 \%$ quantile

\begin{tabular}{lcccccccccc} 
IC \# & 1 & 2 & 3 & 4 & 5 & 6 & 7 & 8 & 9 & 10 \\
\hline Cluster 1 & 4 & 9 & $\mathbf{1 6}$ & 1 & 8 & 7 & 23 & 1 & $\mathbf{2 7}$ & 8 \\
Cluster 2 & 23 & 26 & $\mathbf{2 6}$ & 8 & 30 & 19 & 20 & 11 & $\mathbf{1 7}$ & 20 \\
Cluster 3 & 10 & 5 & 3 & $\mathbf{2 4}$ & 3 & 15 & 6 & 18 & 2 & 12 \\
Cluster 4 & 11 & 7 & 5 & 3 & 6 & 4 & 0 & 13 & 4 & 9 \\
Cluster 5 & 2 & 3 & 0 & $\mathbf{1 4}$ & 3 & 5 & 1 & 7 & 0 & 1 \\
\hline
\end{tabular}

(b) above the $95 \%$ quantile

\begin{tabular}{lcccccccccc} 
IC \# & 1 & 2 & 3 & 4 & 5 & 6 & 7 & 8 & 9 & 10 \\
\hline Cluster 1 & 16 & 13 & 0 & $\mathbf{2 3}$ & 9 & 10 & 1 & 26 & $\mathbf{1 3}$ & 6 \\
Cluster 2 & 17 & 24 & 10 & $\mathbf{1 6}$ & 21 & 19 & 24 & 22 & $\mathbf{2 8}$ & 19 \\
Cluster 3 & 4 & 3 & $\mathbf{1 4}$ & 7 & 7 & 11 & 13 & 2 & 3 & 6 \\
Cluster 4 & 13 & 8 & $\mathbf{8}$ & 3 & 12 & 3 & 7 & 0 & 5 & 19 \\
Cluster 5 & 0 & 2 & $\mathbf{1 8}$ & 1 & 1 & 7 & 5 & 0 & 1 & 0 \\
\hline
\end{tabular}

A close look at IC 3 reveals that the load described by the component responds for every RTP variation, inversely if the weights is positive (above 95\% quantile) or following if the weight is negative (under 5\% quantile). From Table IIa, groups 3 and 4 are the one following the variation of the price. From Table IIb group 6 is the one reacting inversely to every variation of the price.

IC 4 has a pattern which is totally independent from the price. When the weight is positive, it displays an increase of the consumption from 08:00 to $12: 00$. When the weight is negative, it is the opposite, the consumption drops from 08:00 to $12: 00$. This pattern seems to depend on the presence of somebody during the day (positive) or an 'economy setting' (negative).

IC 9 displays a rebound, it anticipates the peak in price (at 07:00) by heating up and using the thermal inertia of the dwelling and cuts heating for the next two hours and half (positive weight) or inversely (negative weight). This is a sign that the controller makes its decision based on information obtained from the day-ahead price or hour-ahead price. The results from Table II clearly shows that groups 6 and 4 are both divided almost into the same proportion between large positive and large negative weights. It is also interesting to note that both groups are equipped with electric heating.

The results shown in Table III are not as clear as the one in Table II but some tendencies can be observed. On IC 3, the most negative weights are mostly from clusters 1 and 2 and the highest positive are from clusters 3,4 and 5 which confirm their active response (on this date). On IC 4, the largest positive weights are from clusters 1 and 2 which have the largest share of standard houses. On the negative side, the clusters 3 and 5 which have the largest shares of summer houses. Clusters 1 and 2 combined are approximately in the same numbers under 5\% and above $95 \%$; it is a consequence of the composition of these clusters which have a majority of households from groups 4 and 6 which presents the same cleavage into half rebound before and half rebound after.

The analysis was also run on the type of housing and only 


\section{Component 3}

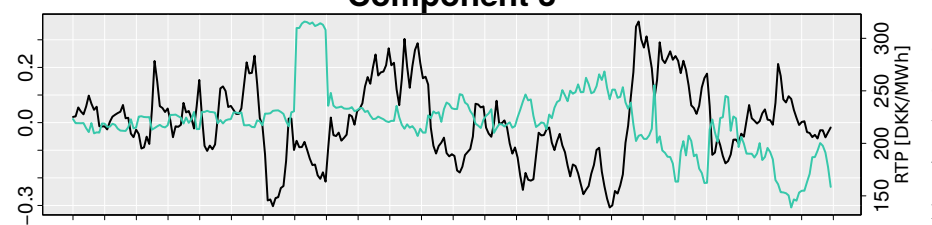

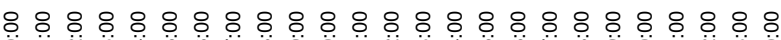

ठ் Component 4

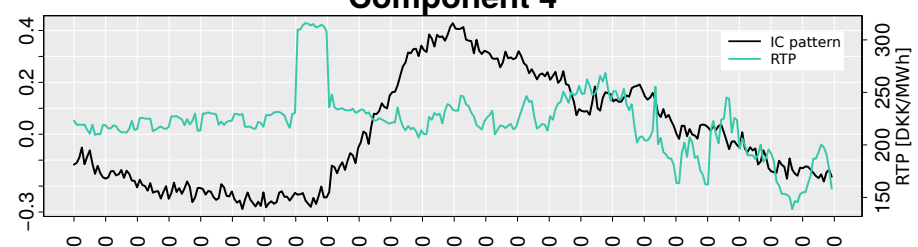

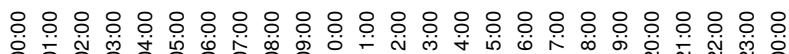
Component 9

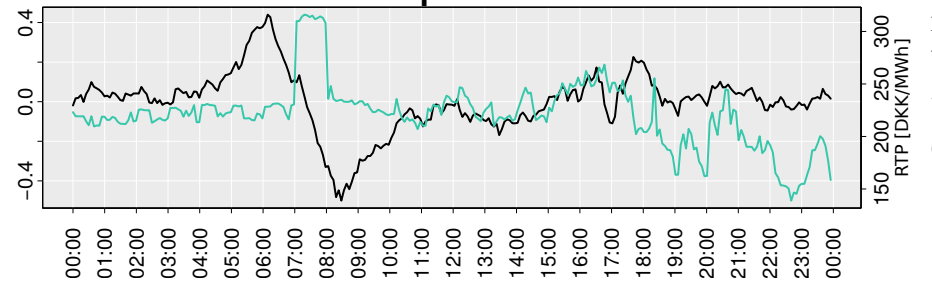

Fig. 5: Representation of the IC number 3, 4 and 9 with the RTP in the same period (06-01-2015)

IC 4 splits clearly between summer houses (negative) and standard houses (positive), which confirms that IC 4 pattern is an 'economy setting'. It also rejects the idea that the type of housing is a factor facilitating the response to a small variation of price, which corresponds to IC 3.

It appears that ICA provides meaningful results when studied in details. The IC 3, 4 and 9 illustrate three different types of observed behaviors, response to RTP (IC 3), setting of the controller by the user (IC 4), and anticipation of a price variation due to controller scheduling (IC 9). The other ICs expose other various behaviors.

\section{CONClusion AND Future Work}

The results from the clustering (Section IV-A) combined with the results from the FIR analysis (Section IV-B) and the ones from the ICA (Section IV-C) investigate the problem from 2 different angles. The first one intends to give an overall estimation of the average response of the households along the heating season as well as the variability by categorizing them and quantifying the response of each cluster. The second aims at characterizing the response as a linear combination of independent signals (i.e. IC). This way the details of the response of each household can be comprehended as the sum of different load behaviors resulting from interactions with the inhabitants, heating scheduling or response to price incentives. Combining this information provides valuable insights for a system operator who might want to activate different clusters at different times to deliver a more finely-tuned response than activating a single cluster would achieve.
The ICA has shown promising results for this specific application and it could theoretically be used to create specific aggregated responses by sampling households with specific weights. The FIR analysis can actually be used as a validation tool after sampling process, as it uses historical data and models the response of a pool of households. Subsequently, different prices could be sent to households to obtain a predefined aggregated load. This approach assumes a certain stationarity of the time-series which is known to not be true when looking at single consumption time-series, but which can be an acceptable assumption when looking at aggregated consumption time-series. In the continuity of this idea, a unit commitment model with different prices per subgroup of households could be implemented to evaluate the benefit of broadcasting different prices.

The drawback of ICA is that is does not produce good results on long time-series. An extensions of this work could be to implement an online ICA or a similar method which would provide results on the entire period instead of only one day.

\section{ACKNOWLEDGMENT}

The authors thank our EcoGrid EU partners for supporting this work, as well as the EUDP for funding through the EnergyLab Nordhavn project (EUDP 64015-0055).

\section{REFERENCES}

[1] P. Palensky and D. Dietrich, "Demand side management: Demand response, intelligent energy systems, and smart loads," IEEE transactions on industrial informatics, vol. 7, no. 3, pp. 381-388, 2011.

[2] P. Siano, "Demand response and smart grids - A survey," Renewable and Sustainable Energy Reviews, vol. 30, pp. 461-478, 2014.

[3] V. Giordano, F. Gangale, and Others, "Smart Grid projects in Europe: lessons learned and current developments," JRC Reference Reports, Publications Office of the European Union, 2011.

[4] N. O'Connell, P. Pinson, H. Madsen, and M. O'Malley, "Benefits and Challenges of Demand Response: A Critical Review," Renewable and Sustainable Energy Reviews, vol. 39, pp. 686-699, 2013.

[5] J. L. Mathieu, P. N. Price, S. Kiliccote, and M. A. Piette, "Quantifying changes in building electricity use, with application to demand response," IEEE Transactions on Smart Grid, vol. 2, no. 3, pp. 507518, 2011.

[6] G. Le Ray, E. M. Larsen, and P. Pinson, "Evaluating price-based demand response in practice - with application to the EcoGrid EU Experiment," IEEE Transactions on Smart Grid, pp. 1-1, 2016.

[7] P. Lund, P. Nyeng, R. N. Grandal, S. H. Sørensen, and Others, "Deliverable 6.7 Overall evaluation and conclusion integration of DER and DR,' EcoGrid EU Final Report, Tech. Rep., 2016.

[8] Y. Ding, S. Pineda, P. Nyeng, J. Østergaard, E. M. Larsen, and Q. Wu, "Real-time market concept architecture for EcoGrid EU - A prototype for European smart grids," IEEE Transactions on Smart Grid, vol. 4, no. 4, pp. 2006-2016, 2013.

[9] E. M. Larsen, P. Pinson, F. Leimgruber, and F. Judex, "Demand response evaluation and forecasting - methods and results from the EcoGrid EU experiment," Sustainable Energy, Grids and Networks, in press.

[10] P. Montero and J. Vilar, "TSclust: An R Package for Time Series Clustering,' JSS Journal of Statistical Software, vol. 62, no. 1, pp. 1-43, 2014.

[11] G. James, D. Witten, T. Hastie, and R. Tibishirani, An Introduction to Statistical Learning, 2013.

[12] A. Hyvärinen and E. Oja, "Independent component analysis: Algorithms and applications," pp. 411-430, 2000. 